EPiC Series in Engineering
Volume 3, 2018, Pages 1584-1588
HIC 2018. 13th International
Conference on Hydroinformatics

\title{
Study on optimization of the operation of dams using Ensemble Prediction and a Distributed Rainfall-Runoff Model
}

\author{
Satoru Oishi ${ }^{1}$ and Toshihiko Tahara ${ }^{2}$ and Mariko Ogawa ${ }^{1}$ \\ ${ }^{1}$ Research Center for Urban Safety and Security, Kobe University, Kobe 657-8501, Japan \\ ${ }^{2}$ Graduate School of Engineering, Kobe University, Kobe 657-8501, Japan \\ tetsu@phoenix.kobe-u.ac.jp
}

\begin{abstract}
Recently, heavy rain by typhoon increases risk of disaster everywhere in Japan. There has been considerable interest to improve the flood control function of dams by prior releasing because an action plan was enacted to fully use the existing hydraulic structures to prevent flood disasters. Carrying out prior releasing has a risk in terms of water supply purpose, in other words, it may cause artificial drought. Therefore, it should be taken into consideration that releasing larger amount of water than rain gives water shortage. In the present study, we suggested the method of dam operation based on rainfall forecast including prior releasing considering the risk in terms of water supply purpose. Concretely, first, we investigated that it could estimate the accuracy of forecasted accumulated rainfall based on Global Spectral Model (GSM) by adding the information of spreads calculated by Weekly Ensemble Prediction System (WEPS) in the Yodo river basin. Second, accumulated rainfall based on GSM errors using gamma distribution was analyzed. Third, the method of dam operation based on rainfall forecast including prior releasing was applied to past examples and the effect was verified. As a result, peak discharge in Hirakata point was reduced than normal operation in case of rainfall prediction was accurate.
\end{abstract}

\section{Introduction}

Recently, localized heavy rains and typhoons have caused serious damage everywhere in Japan. Previously, such flood related disasters were assumed to give damage only in the central and southern part of Japan. Even in the central and southern part of Japan, amount of rain becomes bigger than that between 1970s and 1990s. Therefore, Japanese Ministry of Land Infrastructure Transport and Tourism (MLIT) established the urgent action plan against heavy rain disaster in 2004. The urgent action plan includes prior releasing. Prior releasing is defined as releasing stored water from dam 
reservoirs before heavy rainfall with considering the impact to the water supply purpose of dam reservoirs such as irrigation, industry and domestic water. The amount of water released by prior releasing should be recovered by inflow discharge to dam reservoir in order to match the water supply purposes of dam. The implementation of prior releasing requires well accurate quantitative precipitation forecasting (QPF) with enough lead-time because Japanese typical river has just two or three hours of responding time between rainfall and inflow into dam reservoirs.

In the present study, we propose real-time flood control operation method for dams in a river system in Japan using prior releasing with considering the accuracy of QPF.

\section{Study methods}

The Yodo river system passing through Osaka, Kyoto, Hyogo, Shiga and Mie prefectures is the target river of the present study. The length is $75 \mathrm{~km}$ and the area is $8240 \mathrm{~km} 2$. The population in the river basin is approximately 12 million and it supplies water for approximately 17 million population. The river system has seven dams and one weir. Therefore, both flood control and water supply are very important for managing the Yodo river system.

In the present study, we investigated the accuracy of QPF by using the result of ensemble weather prediction issued by Japan Meteorological Agency (JMA) to avoid negative impact of prior releasing to water supply purpose. Then we propose real-time flood control operation method for dams with ensemble weather prediction and real-time monitoring.

The ensemble prediction is a weather prediction method in which initial perturbation expands through non-linear numerical weather prediction time integration for showing their uncertainty. The expanded perturbation through the numerical weather prediction is analyzed as spread of ensemble prediction. Therefore, we investigated the relationship between spread of ensemble prediction, ensemble mean and the correctness of the QPF. Then, the quantitative error estimation method of QPF is applied to show effectiveness of ensemble weather prediction.

\section{Estimation of Uncertainty of Cumulative Rainfall Amount by using Ensemble Weather Prediction}

Quantitative weather prediction is necessary for prior releasing because responding time between rainfall and inflow of dam reservoir is short in the target basin. The result of numerical weather prediction model is suitable for quantitative weather prediction. JMA is operating Global Spectrum Model (GSM) and Meso Scale Model (MSM) for such purpose. GSM is more appropriate for the target basin because it has longer lead time 84hours than MSM, 39hours. JMA also operates Weekly Ensemble Prediction System (WEPS) (Sakai, 2008) for showing stochastic aspects of weather forecasting. We assumed that WEPS represented the uncertainty of prediction of GSM because they have same basic equations. Therefore, we use the spread of some indexes using result of WEPS. The indexes investigated in the present study are position (longitude, latitude and distance from reservoir) of typhoon centre, surface pressure, wind velocity and direction. The relationship between the spread of these indexes and accuracy of the forecasted rainfall amount by GSM is investigated to select the better index showing worse QPF from GSM. Moreover, the mean of predicted rainfall by WEPS is also investigated to get better QPF from GSM.

The investigation has been conducted with 58 typhoons passing the area (30-40N, 125-145E) in 2008 to 2015 . The predictions of three days, two days and one day before the passing the area have been used when $100 \mathrm{~mm}$ of rainfall and more is expected in each prediction. 
Study on Optimization of the Operation of Dams Using Ensemble Prediction and a ... S. Oishi et al.

The investigation shows that bigger spread of surface pressure of WEPS implies over estimation of QPF by GSM. Here, over estimation means prediction estimates bigger amount of rainfall than actual and it results insufficient water recover after prior releasing. Moreover, bigger mean of predicted rainfall by WEPS implies the certainty of QPF by GSM that means we can expect enough amount of water recovery after prior releasing when mean of predicted rainfall by WEPS is more than threshold. The combination of threshold of WEPS pressure spread and WEPS mean rainfall is shown in Table 1.

After selecting the GSM prediction for using prior releasing, we analysed the error of accumulation of QPF by GSM by referring Mitsuichi et al. (Mitsuishi, Sumi, Ozeki, S, \& Sakai, 2011) which uses gamma distribution for probable density function (PDF). Table 2 shows the ratio of QPF whose accumulation is less than observed rainfall amount and it shows the effectiveness of selection using WEPS indexes mentioned above. WEPS indexes selection reduces the ratio for 12 to $37 \%$. Especially, it reduces the ratio to $10-15 \%$ from $35-46 \%$ at Shorenji, Hinachi and Takayama dams.

\begin{tabular}{lccc}
\hline Name of dams & $\begin{array}{c}\text { normalised WEPS } \\
\text { pressure spread }\end{array}$ & & mean rainfall amount by WEPS \\
\hline Hiyoshi & $>0.15$ & and & $<120 \mathrm{~mm}$ \\
Amagase & $>0.15$ & and & $<150 \mathrm{~mm}$ \\
Murou & $>0.15$ & or & $<140 \mathrm{~mm}$ \\
Shorenji & $>0.2$ & or & $<100 \mathrm{~mm}$ \\
Hinachi & $>0.2$ & or & $<100 \mathrm{~mm}$ \\
Takayama & $>0.15$ & or & $<130 \mathrm{~mm}$ \\
Nunome & $>0.15$ & or & $<140 \mathrm{~mm}$ \\
\hline
\end{tabular}

Table 1 : Combination of indexes of WEPS showing uncertain prediction of QPF by GSM.

\begin{tabular}{lcc}
\hline Name of dams & All typhoons & $\begin{array}{c}\text { Typhoons selected by using WEPS } \\
\text { indexes }\end{array}$ \\
\hline Hiyoshi & $45 \%$ & $33 \%$ \\
Amagase & $60 \%$ & $46 \%$ \\
Murou & $51 \%$ & $22 \%$ \\
Shorenji & $35 \%$ & $11 \%$ \\
Hinachi & $35 \%$ & $10 \%$ \\
Takayama & $46 \%$ & $15 \%$ \\
Nunome & $68 \%$ & $31 \%$ \\
\hline
\end{tabular}

Table 2 : Ratio of QPF accumulation less than observed rainfall amount.

\section{Proposing Real-time Flood Control Operation for Dams using Precipitation Forecasting}

We propose a strategy to release water from reservoir with considering the maximum estimated discharge of inflow $Q_{\max }$ into dam reservoir and minimum estimated discharge $Q_{\min }$ of inflow. The strategy is explained in the following steps. 
- When observed inflow at one unit time previous ( $\mathrm{t}-1)$ is less than Qmin, releasing water discharge should be reduced to base flow discharge to recover water for the other purpose than flood control.

- When observed inflow at one unit time previous (t-1) is larger than Qmax, releasing water discharge should follow the flood control rule.

- When observed inflow at one unit time previous (t-1) is larger than Qmin and less than Qmax, releasing water discharge is decided as the following equation,

$$
Q_{\text {out }}(t)=Q_{f c}(t)-\left\{Q_{\max }(t-1)-Q_{\text {in }}(t-1)\right\}
$$

where, $Q_{o u t}(t)$ : proposed discharge at time $t, Q_{f c}(t)$ : discharge of flood control rule, $Q_{\max }(t-1)$ : the maximum estimated discharge of inflow at one unit time previous $(t-1), Q_{i n}(t-1)$ : observed dam reservoir inflow at one unit time previous $(t-1)$.
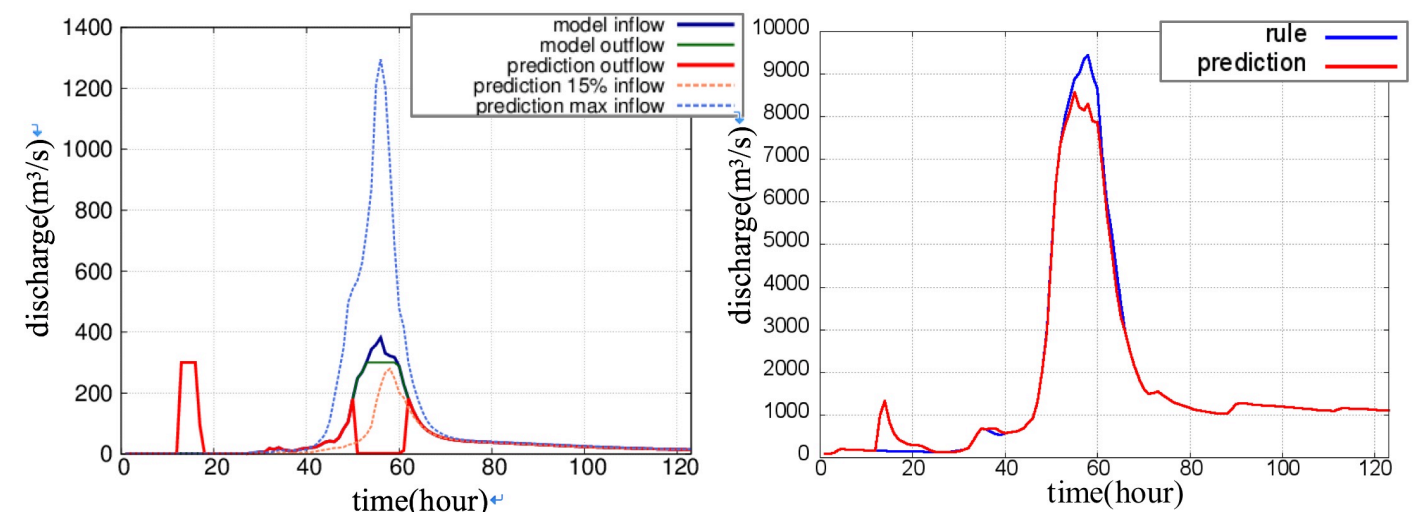

Figure 1: Result of proposed strategy $\mathrm{u} / \mathrm{sing}$ prior releasing at Hinachi dam (left) and Hirakata flood control point (right).

We have applied the proposed strategy to flood caused by Typhoon No. 18 in 2013. Figure 1 shows the discharge of inflow into dam reservoir and proposed outflow discharge from dam in the left-hand side, comparison of discharge at a flood control point in the lower basin in the Yodo river (Hirakata point) in the right-hand side. In the left-hand side, model inflow means discharge into Hinachi dam reservoir; model outflow means outflow discharge from Hinachi dam by following flood control rule without prior releasing; prediction outflow means proposed outflow discharge from Hinachi dam to conduct prior releasing which released water from irrigation capacity more than twenty hours before flood came; prediction $15 \%$ inflow means the minimum estimated discharge of inflow; prediction max inflow means the maximum estimated discharge of inflow both calculated by WEPS rainfall forecast. The difference between peak outflow discharge following flood control rule and proposed outflow discharge was $298 \mathrm{~m}^{3} / \mathrm{s}$. The proposed strategy has been applied all the seven dams in the Yodo river system and it reduced $1147 \mathrm{~m}^{3} / \mathrm{s}$ of discharge at flood control point. The amount of discharge is equivalent to the $0.49 \mathrm{~m}$ water level difference.

\section{Conclusion}

We proposed the method to recognize uncertainty of QPF of GSM by using WEPS pressure spread and WEPS mean rainfall as well as the strategy to decide release water discharge from dams 
Study on Optimization of the Operation of Dams Using Ensemble Prediction and a ... S. Oishi et al.

using the maximum estimated discharge of inflow and the minimum estimate discharge both calculated by WEPS rainfall forecast. The proposed method to recognize uncertainty of QPF reduced ratio of QPF of GSM whose accumulation is less than observed rainfall amount. Especially, it reduced to $10-15 \%$ at three dams in the Yodo river basin. The proposed strategy has been applied to the typhoon case in 2013 showing effectiveness of the strategy to reduce peak discharge from dam reservoir as well as discharge at the Hirakata flood control point.

\section{References}

Mitsuishi, S., Sumi, T., Ozeki, T., S, \& Sakai, R. (2011). New Weekly Ensemble Weather Forecasting System of JMA. Dam Engineering 21(4) (in Japanese), 242-250.

Sakai, R. (2008). New Weekly Ensemble Weather Forecating System of JMA. Tenki 55(6), 515-520. 